\title{
THE SET OF IRREDUCIBLE OPERATORS IS DENSE
}

HEYDAR RADJAVI AND PETER ROSENTHAL

Let $\mathfrak{H C}$ be a separable (finite or infinite-dimensional) complex Hilbert space. P. R. Halmos [1] has shown that the set of irreducible operators on $\mathcal{H C}$, (i.e., operators with no nontrivial reducing subspaces), is uniformly dense in the space of bounded operators on $3 C$. In this note we give a very simple proof of Halmos's theorem.

Let $A$ be any bounded operator on $\mathcal{F}$ and let $\epsilon>0$. By the spectral theorem there exists a Hermitian operator $D$ whose matrix is diagonal with respect to an o.n. basis $\left\{e_{n}\right\}$ such that

$$
\left\|D-\left(A+A^{*}\right) / 2\right\|<\frac{\epsilon}{4} .
$$

Then there is a Hermitian operator $D_{1}$ diagonal with respect to $\left\{e_{n}\right\}$ such that all the eigenvalues of $D_{1}$ are distinct and $\left\|D-D_{1}\right\|<\epsilon / 4$. Now let $D_{2}$ be any Hermitian operator within $\epsilon / 2$ of $\left(A-A^{*}\right) / 2 i$ whose matrix with respect to $\left\{e_{n}\right\}$ has all entries different from 0 ; (such operators obviously exist in profusion). Then the operator $D_{1}+i D_{2}$ is within $\epsilon$ of $A$. Also $D_{1}+i D_{2}$ is irreducible, since the invariant subspaces of $D_{1}$ are the subspaces spanned by subcollections of $\left\{e_{n}\right\}$, and none of these are invariant under $D_{2}$ except $\{0\}$ and $\mathfrak{H}$.

\section{REFERENCE}

1. P. R. Halmos, Irreducible operators, Michigan Math. J. 15 (1968), 215-223.

UNIVERSITY OF TORONTO

Received by the editors September 12, 1968. 